\title{
Polymorphism of Theophylline Metabolism in Man
}

Carol A. Miller, Leslie B. Slusher, and Elliot S. Vesell

Department of Pharmacology, The Pennsylvania State University College of Medicine, Hershey, Pennsylvania 17033

\begin{abstract}
To determine whether genetic mechanisms control large interindividual variations in theophylline elimination in normal uninduced human subjects, and, if so, to test the possibility that these genetic factors are transmitted as a simple Mendelian trait, theophylline was administered to 79 unrelated adults, six sets of monozygotic twins, six sets of dizygotic twins, and six two-generation families. Thereafter, in urine collected from each subject at regular intervals for $48 \mathrm{~h}$, concentrations of theophylline and its three principal metabolites were measured and rate constants of formation of these metabolites calculated.

The twin study, designed to determine the relative contributions of genetic and environmental factors to large interindividual variation in theophylline elimination, revealed predominantly genetic control. Values for this genetic component, designated heritability $\left(\mathrm{H}_{1}{ }^{2}\right)$, of interindividual variation in rate constants of metabolite formation were $0.61,0.84$, and 0.95 for 3-methylxanthine, 1-methyluric acid, and 1,3-dimethyluric acid, respectively. $\mathrm{H}_{1}{ }^{2}$ for the overall theophylline elimination rate constant $\left(k_{\mathrm{el}}\right)$ was lower $(0.34)$.

In the 79 unrelated adults, each distribution curve for rate constants of formation of each theophylline metabolite appeared to be trimodal. By contrast, the distribution curve for the overall theophylline elimination rate constant appeared to be either unimodal or bimodal. The extent of interindividual variation was fourfold for theophylline $k_{\mathrm{el}}$ and 6-8-fold for the three principal metabolites. High correlations among the three rate constants in individual subjects suggested their regulation by a single shared factor.

In six families carefully selected to be under near basal environmental conditions so that hepatic theophylline metabolism of each family member would be neither markedly induced nor inhibited, phenotypes for theophylline metabolite rate constants were assigned. This assignment of phenotype was made by the position of each family member's rate constant on the three distribution curves that were generated from the 79 unrelated subjects. In each family, pedigree analysis of the three phenotypes for each rate constant was consistent with their control by two alleles at a single genetic locus and with autosomal codominant transmission. Frequencies of the two alleles at each genetic locus controlling rate constants of formation of theophylline metabolites were similar (p $=0.49,0.53$, and 0.52 ). In the three families studied with antipyrine (AP) as well as with theophylline, AP $\boldsymbol{k}_{\mathrm{el}}$ correlated $(r \sim 0.7)$ with each rate constant of theophylline metabolite
\end{abstract}

\footnotetext{
Address reprint requests to Dr. Vesell.

Received for publication 25 June 1984 and in revised form 22 January 1985.
}

J. Clin. Invest.

(c) The American Society for Clinical Investigation, Inc. $0021-9738 / 85 / 05 / 1415 / 11 \quad \$ 1.00$

Volume 75, May 1985, 1415-1425 formation, as well as with theophylline $\boldsymbol{k}_{\mathrm{el}}$. While these results are compatible with a common regulatory element in the AP and theophylline polymorphisms, other evidence suggests more than a single genetic polymorphism. This additional evidence includes different gene frequencies for the $\mathrm{AP}(p \sim 0.1)$ and theophylline $(p \sim 0.5)$ polymorphisms, different genotype assignments in several families for some theophylline metabolites, different distribution curves for theophylline $k_{\mathrm{el}}$ from those for the three theophylline metabolites in 79 unrelated subjects, and finally low correlations between AP metabolite rate constants and theophylline metabolite rate constants in the three families receiving both drugs.

\section{Introduction}

Theophylline is commonly used as a bronchodilator and respiratory stimulant in acute and chronic asthma, CheyneStokes respirations, and episodes of apnea/bradycardia in newborns (1). It also serves as an adjunct in the therapy of congestive heart failure and acute pulmonary edema (1). In a particular patient, the therapeutic and toxic properties of theophylline depend on the rate of hepatic theophylline metabolism, since benefits and risks from therapy with theophylline relate directly to serum theophylline concentrations. In each patient, serum theophylline concentrations are functions both of the dose and elimination characteristics of the drug (1). In adults as well as children, large interpatient variations occur in rates of theophylline elimination (2-5). This metabolic individuality of a particular subject has been stated to arise from interactions among genetic, environmental, physiological, and perhaps pathological factors that determine the activity of the hepatic mixed function oxidases that biotransform theophylline (6). Diverse environmental factors are known to influence theophylline clearance (1). Although genetic factors have also been frequently implicated in causing interindividual variations in theophylline metabolism (7-9), evidence to support such a genetic hypothesis has until the present study been notably absent.

The present investigation was designed, therefore, to determine whether genetic mechanisms control these large interindividual variations in theophylline elimination in human subjects and, if they do, to test whether they are transmitted as a Mendelian trait. It was also of interest to determine whether any genetic factor affecting theophylline metabolism was closely related or identical to previously described polymorphisms of hepatic drug oxidation, such as those of debrisoquine (10), mephenytoin (11), and antipyrine (AP) ${ }^{1}(12)$.

1. Abbreviations used in this paper: $\mathrm{AP}$, antipyrine; $\mathrm{DZ}$, dizygotic; $\mathrm{H}^{2}$, heritability; $\mathrm{H}_{1}{ }^{2}, \mathrm{H}_{2}{ }^{2}, \mathrm{H}_{3}{ }^{2}$, and $\mathrm{H}_{4}{ }^{2}$, methods one, two, three, and four for heritability; HPLC, high performance liquid chromatography; $k_{\mathrm{el}}$, overall elimination rate constant; $k_{i}$, rate constant of metabolite formation; 1-MU, 1-methyluric acid; 1,3-MU, 1,3-dimethyluric acid; 3-MX, 3-methylxanthine; MZ, monozygotic; TBHAS, tetrabutyl ammonium hydrogen sulfate. 
The approach used in the present study consists of measuring rate constants of formation of theophylline metabolites in twins, unrelated subjects, and carefully selected families. In studies of normal families, rate constants of theophylline metabolite formation are as close to the primary gene products involved in theophylline metabolism as it is now possible to come short of the ethically unjustifiable liver biopsy. These primary gene products are different molecular forms (isozymes) of hepatic cytochrome P-450.

The 3 theophylline metabolites investigated were 3-methylxanthine (3-MX), 1-methyluric acid (1-MU), and 1,3-dimethyluric acid (1,3-MU). Developmental and enzyme inductive studies suggest that the $\mathrm{N}$-demethylation and 8 -hydroxylation pathways of theophylline metabolism are mediated by two separate isozymes of cytochrome P-450 (3, 13-15).

The rationale for the approach used in the present study derives from the conventional methodology in human genetics of testing a genetic hypothesis by analysis of pedigrees in which phenotypes are assigned according to the place of each family member within distribution curves of data gathered from unrelated subjects. This approach helped to elucidate several pharmacogenetic conditions, including atypical plasma cholinesterase, the acetylation polymorphism, glucose-6-phosphate dehydrogenase deficiency, and the polymorphic oxidations of debrisoquine (10), mephenytoin (11), and AP (12).

\section{Methods}

Selection of subjects. Normal subjects between the ages of 18 and 57 participated (27 from 6 two-generation families, 6 sets of monozygotic (MZ), and 6 sets of dizygotic (DZ) twins, and another 79 who were unrelated). Subjects were recruited from Hershey, PA and vicinity through notices placed in the medical school. They were carefully selected to be under near basal environmental conditions so that their rate of theophylline metabolism would be neither markedly induced nor inhibited. 15 of the 27 family members and several twins and unrelated subjects had previously participated in the AP studies performed in this laboratory $(12,16)$. No subject had a history of serious illness or drug allergy, consumed any medications or ethanol regularly, smoked tobacco, or was chronically exposed to inducing or inhibiting chemicals.

Three of the six families were available from the 13 families previously recruited in this laboratory to identify the polymorphism affecting AP metabolism (12) because these families (numbered family 2,11 , and 13 in that study) offered an opportunity to trace transmission of the less frequently occurring AP allele. Evidence from other laboratories had indicated a positive correlation within subjects between rates of theophylline and AP metabolism (17-19). Therefore, only the most informative families from our AP study (12) were used, since the present study attempted to determine whether one genetic locus might control a single cytochrome P-450 isozyme that regulates both AP and theophylline metabolism. 79 unrelated individuals were deemed sufficient to uncover a commonly occurring polymorphism, i.e., one in which $\mathrm{q}>0.1$. The debrisoquine polymorphism was discovered using a similar number of subjects (10). Under a monogenic, two allele system a value of 0.1 for $q$ derives from calculations of the number of individuals expected $\left(\mathrm{p}^{2}, 2 \mathrm{pq}\right.$, and $\left.\mathrm{q}^{2}\right)$ with a phenotype corresponding to homozygosity for the $p$ allele, heterozygosity, and homozygosity for the $q$ allele, respectively, when different theoretical frequencies were assigned for alleles $p$ and $q$.

Materials. Chemicals used in the chromatographic analysis were 1,3-MU and 3-MX from Fluka AG; 1-MU from Adams Chemical, Round Lake, IL; theophylline from ICN Pharmaceuticals, Inc., Irvine, $\mathrm{CA}$; and $\beta$-hydroxyethyltheophylline from Sigma Chemical Co., St. Louis, MO. All chemicals used in the extraction procedure were either analytical or high performance liquid chromatography (HPLC) grade.
All organic solvents used in the chromatographic procedure were HPLC grade.

Sample collection. Subjects abstained from all medications and alcoholic beverages for $2 \mathrm{wk}$ preceding and during the study. For $3 \mathrm{~d}$ before as well as during the study, subjects avoided foods and beverages containing methylxanthines. All were required to fast from solid foods for $8 \mathrm{~h}$ before and $4 \mathrm{~h}$ after receiving theophylline.

Two single-dose studies (protocols 1 and 2) were performed as follows: protocol $1-13$ subjects reported at 0730 at which time a teflon intravenous catheter with a heparin well was placed in the forearm for removal of blood samples. At 0800 , blood and urine samples were collected to be assayed as blanks. The subject was then given an oral dose $(5 \mathrm{mg} / \mathrm{kg}$ ) of theophylline (Elixicon by Berlex Laboratories, Wayne, NJ, a theophylline suspension). Thereafter, 5-ml blood samples were drawn and heparinized at 1, 2, 3, 5, 8, 13, 24, 28, 32, 36, and $48 \mathrm{~h}$. Complete urine samples were collected at 4-h intervals for the first $48 \mathrm{~h}$, then from 48 to $54 \mathrm{~h}, 54$ to $60 \mathrm{~h}$, and 60 to $72 \mathrm{~h} .10$ of the subjects repeated the above study $2 \mathrm{wk}$ later.

In addition to providing blood, three subjects collected saliva samples, which were stimulated by chewing parafilm, at 4, 8, 12, 16, $20,24,28,32$, and $36 \mathrm{~h}$ after drug administration. These three subjects did not take theophylline again. Protocol 2-the remainder of the subjects participated in this protocol. At 0800 , after an 8 -h fast, all subjects received an oral dose $(5 \mathrm{mg} / \mathrm{kg}$ ) of theophylline (Elixicon, by Berlex Laboratories). Saliva samples, stimulated by chewing parafilm, were collected at $2,4,6,10,16,20,24$, and $28 \mathrm{~h}$ after theophylline. Complete urine samples were collected at 4-h intervals for the first 24 $h$, then at 6-h intervals for the second $24 \mathrm{~h}$.

All samples were refrigerated after collection. Blood samples were centrifuged at $2,000 \mathrm{~g}$ for $10 \mathrm{~min}$ to obtain plasma. The volume of urine was measured and aliquots saved for subsequent analysis. All samples were frozen at $-20^{\circ} \mathrm{C}$ until assayed. All samples were assayed within 2 mo (20).

Assay procedure. Theophylline and its major metabolites, 3-MX, 1-MU, and 1,3-MU, were extracted from blood, saliva, and urine samples according to the method of Muir et al. (21) with the following modifications: (1) $1 \mathrm{ml}$ of urine was extracted instead of $0.5 \mathrm{ml}$; (2) $0.2 \mathrm{ml}$ of $0.5 \mathrm{M}$ tetrabutyl ammonium hydrogen sulfate (TBAHS) solution was substituted for $0.5 \mathrm{ml}$ of $0.1 \mathrm{M}$ TBAHS; (3) the extraction mixture, ethylacetate/chloroform/isopropanol, was 40:40:20, vol/vol; (4) the reconstituting buffers contained $0.05 \mathrm{M}$ sodium acetate instead of $0.01 \mathrm{M}$. Only $0.2 \mathrm{ml}$ of each solution was used to reconstitute samples.

Aliquots of saliva $(1.0 \mathrm{ml})$ or plasma $(0.5 \mathrm{ml})$ were extracted as described for urine except only $0.1 \mathrm{ml}$ of the internal standard solution and only $0.1 \mathrm{ml}$ of each reconstituting solution were used. All glassware used in the extractions was treated with a silicone preparation (Prosil-28).

Concentrations of theophylline and its three main metabolites were measured by reversed-phase HPLC. Either $25 \mu$ l of the urine extract or $80 \mu \mathrm{l}$ of the saliva or plasma extract was injected onto a RadialPAK $\mathrm{C}_{18}$ cartridge $(5 \mu, 8 \mathrm{~mm} \times 10 \mathrm{~cm})$ in series with a $\mathrm{C}_{18}$ RCSS Guard-PAK in an RCM-100 radial compression module (Waters Assoc., Milford, MA) via a model U6K universal injector (Waters Assoc.). A model 45 solvent delivery system (Waters Assoc.) was used with a model 440 absorbance detector (Waters Assoc.) at $280 \mathrm{~nm}$. The mobile phase consisted of $5 \mathrm{mM}$ TBAHS and $10 \mathrm{mM}$ sodium acetate buffer with $6.5 \%$ acetonitrile at ambient temperature with an apparent $\mathrm{pH}$ of 4.77 (flow rate, $2.0 \mathrm{ml} / \mathrm{min}$ ). Retention times for 3-MX, 1-MU, 1,3-MU, theophylline, and internal standard were $2.5,3.2,4.0,6.2$, and $7.3 \mathrm{~min}$, respectively. Calibration curves were obtained from blank plasma, saliva, and urine samples spiked with standards of theophylline and its three main metabolites. The standards were dissolved in 2.5 $\mathrm{ml}$ of $1 \mathrm{~N} \mathrm{NaOH}$ before spiking the blank plasma, saliva, and urine samples. Standard curves were linear in urine for all compounds up to $200 \mu \mathrm{g} / \mathrm{ml}$, with sensitivity being $\sim 1 \mu \mathrm{g} / \mathrm{ml}$. Plasma and saliva standard curves were linear up to $20 \mu \mathrm{g} / \mathrm{ml}$, with sensitivity being $\sim 0.2 \mu \mathrm{g} / \mathrm{ml}$ for parent drug and its metabolites. To measure the 
amount of theophylline and its main metabolites in specimens of plasma and urine, peak area ratios of theophylline and its metabolites relative to the internal standard were obtained in each chromatogram by use of a 3390 A reporting integrator (Hewlett-Packard Co., Rockville, $\mathrm{MD}$ ).

Because metabolite concentrations in blood were below assay sensitivity in the unrelated volunteers studied using protocol 1 , only saliva samples were collected from subjects participating in protocol 2. Saliva samples to measure theophylline concentrations only were prepared for HPLC analysis using the following modified acetonitrile extraction procedure (22). A 10- $\mu$ l aliquot of internal standard, $2 \mathrm{ml}$ of acetonitrile, and $0.5 \mathrm{ml}$ of saliva which had been centrifuged for 20 $\min$ at $2,000 \mathrm{~g}$ were vortexed $15 \mathrm{~s}$ and centrifuged for $15 \mathrm{~min}$ at 2,000 $g$. The supernatant layer was decanted and evaporated at $55^{\circ} \mathrm{C}$ under nitrogen. The residue was reconstituted by first adding $100 \mu \mathrm{l}$ of 0.05

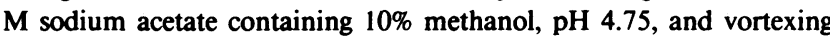
for $60 \mathrm{~s}$, and then adding $100 \mu \mathrm{l}$ of $0.05 \mathrm{M}$ sodium acetate with 0.5 $M$ TBAHS, pH 4.75, and vortexing again for $60 \mathrm{~s}$. A $100-\mu \mathrm{l}$ vol was injected into the HPLC. Saliva standard curves for theophylline were linear up to $20 \mu \mathrm{g} / \mathrm{ml}$.

Pharmacokinetic analysis. Theophylline, when administered at a sub-therapeutic dose $(5 \mathrm{mg} / \mathrm{kg})$, and its three main metabolites, conform to an open one-compartment model with parallel, simultaneously occurring, first-order reactions in which excretion is faster than metabolite formation (23-27). Kinetic data on theophylline elimination were analyzed by two different methods, the Rate method and the SigmaMinus method described in detail elsewhere $(16,26,27)$.

Data analysis for reproducibility. A two-tailed paired $t$ test was performed to evaluate reproducibility of the rate constants of metabolite formation within an individual. This test is appropriate because it estimates both quantity and direction of change.

Genetic analysis of twin data. To estimate the relative contributions of genetic and environmental factors to interindividual variations in the kinetic measurements, heritability $\left(\mathrm{H}^{2}\right)$ was calculated by several methods (28-30). The subscript denotes the method. One approach $\left(H_{1}{ }^{2}\right)$ uses the following equation: $H_{1}{ }^{2}=\left(V_{D Z}-V_{M Z}\right) /\left(V_{D Z}\right)$, in which $V_{M Z}$ and $V_{D Z}$ represent variance among $M Z$ and $D Z$ twins, respectively. Variance was calculated as follows: $\mathbf{V}=\left[\Sigma(\text { difference between twins })^{2}\right]$ / $2 N$, in which $N$ is the number of twin pairs of the same zygosity. This equation permits a range of values from zero (negligible genetic control over variation) to one (virtually complete hereditary influence).

The second method $\left(\mathrm{H}_{2}{ }^{2}\right)$ uses controls (CP) generated by scrambling twin pairs (30) and calculates $\mathbf{H}_{2}{ }^{2}$ as follows: $\mathbf{H}_{2}{ }^{2}=\left(V_{C P}-V_{M z}\right) /\left(V_{C P}\right)$. Other estimates $\left(\mathrm{H}_{3}{ }^{2}\right.$ and $\left.\mathrm{H}_{4}{ }^{2}\right)$ of heritability, based on intraclass correlation coefficients, are: $\mathrm{H}_{3}{ }^{2}=2\left(r_{\mathrm{MZ}}-r_{\mathrm{DZ}}\right)$ and $\mathrm{H}_{4}{ }^{2}=\left(r_{\mathrm{MZ}}\right.$ $\left.-r_{\mathrm{DZ}}\right) /\left(1-r_{\mathrm{DZ}}\right)$, where $r_{\mathrm{MZ}}$ and $r_{\mathrm{DZ}}$ are the intraclass correlation coefficients for $\mathrm{MZ}$ and $\mathrm{DZ}$ twins, respectively. Intraclass correlation coefficients were calculated using the following equation $(31): r_{1}=(2 \Sigma$ $\left.[y-y]\left[y^{\prime}-y^{\prime}\right]\right) /\left(\Sigma[y-y]^{2}\left[y^{\prime}-y^{\prime}\right]^{2}\right)$, where $y$ and $y^{\prime}$ are the values of the two variables thought of as a sample from the population of all such pairs. Intraclass correlation coefficients were also compared for twins living in the same household and twins living in different households, both regardless of zygosity.

Distribution curves and probit analysis. Distribution curves were constructed for all rate constants from all unrelated individuals. Probit analysis (32) was used to attempt to identify modes and antimodes of these curves. The probit, $y$, is defined as the normal equivalent deviation, $(x-\mu) /(\sigma)$, increased by 5 , where $x$ is each data point, and $\mu$ and $\sigma$ are the population mean and standard deviation, respectively. Thus: $y=5+(x-\mu) /(\sigma)$. Regression lines formed when probits were plotted against $\log$ rate constants suggested inflection points where antimodes could be postulated to occur in the distribution curves (32). In each probit regression, the position of these inflection points which suggested a line of different slope was determined as follows: an arrow identified the last subject on the slope of a curve, such that the next subject and subsequent subjects belonged on a curve with a different slope. No subject on this new line of different slope returned to the slope of the preceding line.
Pedigree analysis. Pedigree analysis was performed on each of the six families investigated. Phenotypes were determined by each family member's position on the distribution curves constructed from the $\mathbf{7 9}$ unrelated subjects (Figs. 3-5).

Correlations of rate constants within subjects. To elucidate potential relationships among the genetic factors that control the formation of each main theophylline metabolite, correlation coefficients were determined for the different rate constants in all 99 related and unrelated individuals.

Correlations of metabolic data of theophylline and antipyrine in three families. To assess the degree of correlation between rates of formation of AP and theophylline metabolites after each subject received a single oral dose of each drug on two separate occasions, correlation coefficients were determined for all combinations of the different rate constants in the members of the three families who received both drugs.

\section{Results}

After a single oral dose of theophylline, mean $( \pm S D)$ percentage recoveries of unchanged theophylline, 3-MX, 1-MU, and 1,3MU were $10.3 \pm 3.8,10.8 \pm 3.0,17.7 \pm 5.4$, and $38.8 \pm 8.8$, respectively. The total recovery of administered theophylline as urinary theophylline and metabolites was $77.6 \pm 16.3 \%$ (mean \pm SD). These values are consistent with those reported by others $(14,22,33,34)$.

Reproducibility of measurements in a given subject was evaluated by the two-tailed paired $t$ test in the 10 unrelated subjects who received theophylline on two separate occasions. Intraindividual variations in rate constants remained small and did not reach statistical significance $(P>0.05)$, but interindividual variations, even in only 10 subjects, were approximately twofold. Throughout this study, other subjects who had received theophylline on two separate occasions also exhibited high reproducibility.

In samples of plasma obtained from subjects at various times after theophylline administration, concentrations of theophylline metabolites were below assay sensitivity. Decay curves of theophylline in plasma and saliva from individuals who received a single oral dose of theophylline were parallel. Slopes of these curves in plasma and saliva of a subject differed by $<10 \%$. Peak concentrations of theophylline were attained in plasma between 1 and $2 \mathrm{~h}$ after drug administration, with a mean peak theophylline concentration of $9.7 \pm 1.7 \mu \mathrm{g} / \mathrm{ml}$ (mean $\pm \mathrm{SD}$ ).

In most subjects, theophylline metabolite concentrations in urine could not be measured accurately beyond $48 \mathrm{~h}$ after theophylline administration due to the presence in urine of interfering endogenous xanthines and uric acids at concentrations ranging from 2 to $3 \mu \mathrm{g} / \mathrm{ml}$. In all subjects, urinary excretion of 3-MX, 1-MU, and 1,3-MU plotted according to the Rate and Sigma-Minus methods exhibited parallel, firstorder kinetics (Fig. 1). A lag period of 2-4 h occurred between peak plasma theophylline concentrations and peak urinary excretion rates of metabolites.

Estimates of heritability for the overall elimination rate constant $\left(k_{\mathrm{el}}\right)$ and the rate constants for formation of the three major metabolites $\left(k_{i}\right)$ revealed predominantly genetic control over interindividual variation. Values for heritability, $\mathrm{H}_{1}{ }^{2}$, were $0.61,0.84,0.95$, and 0.34 for $k_{3-\mathrm{MU}}, k_{1-\mathrm{MU}}, k_{1,3-\mathrm{MU}}$ and $k_{\mathrm{el}}$, respectively (Table I).

When intraclass correlation coefficients were compared for those twins, regardless of zygosity, who were living in the same 
MZ twins
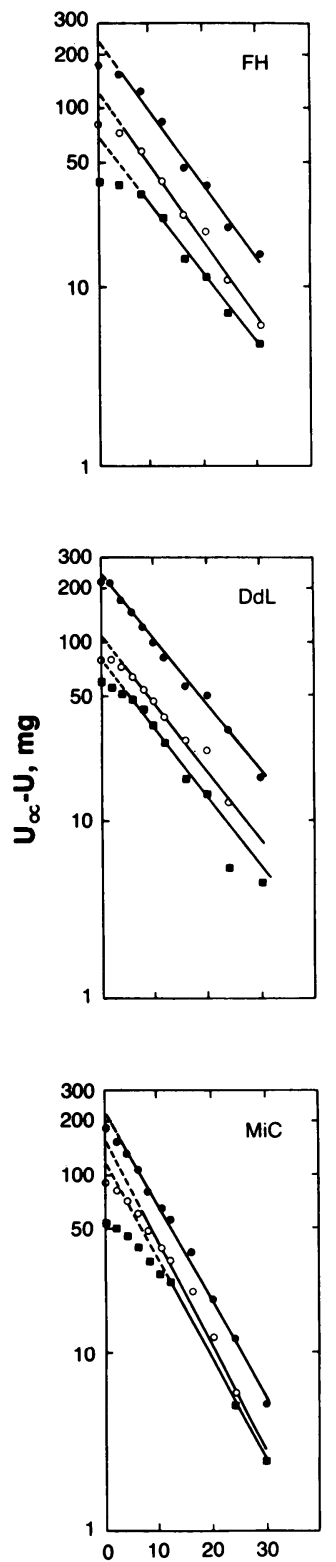
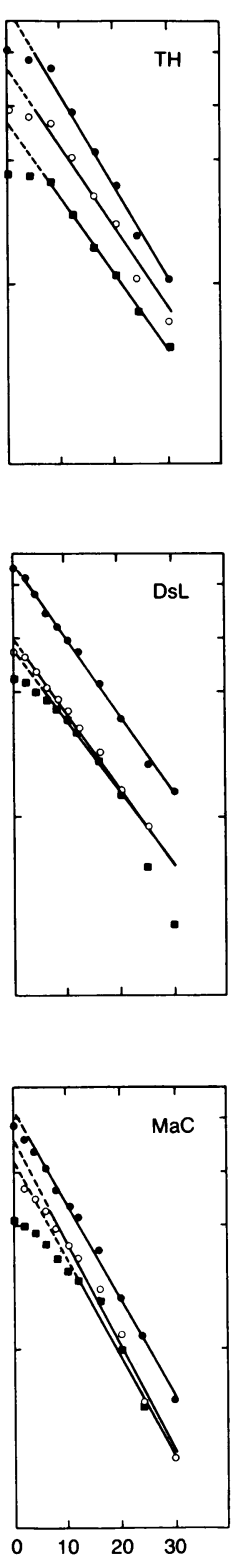

DZ twins
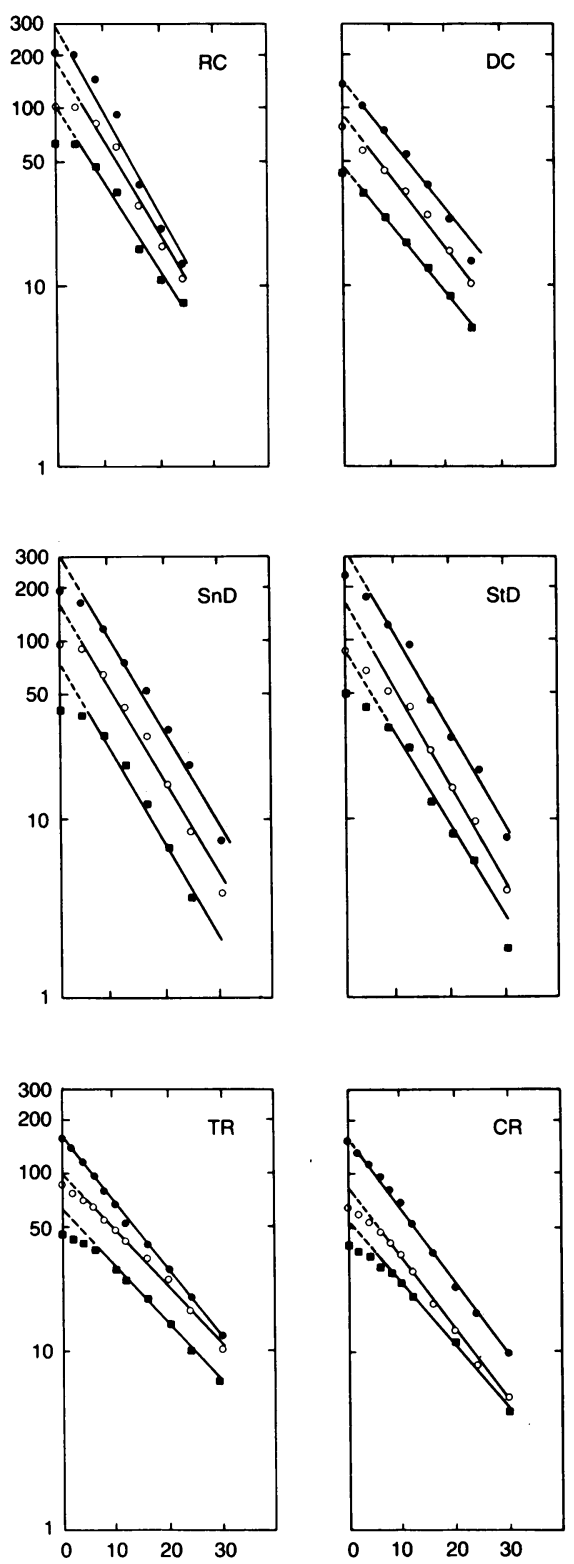

Figure 1. Urinary excretion profiles of 3-MX (घ), 1-MU (0), and 1,3-MU (๑), plotted according to the Sigma-Minus method $(26,27)$, in three representative sets of $\mathrm{MZ}$ and three representative sets of DZ twins. At time 0 each subject took a single oral dose of theophylline $(5 \mathrm{mg}$ / $\mathrm{kg})$. $U_{\infty}$ equals the total amount of the given metabolite excreted. $U$ equals the cumulative amount of a given metabolite excreted up to the time that the particular specimen was obtained.

Time, $\mathbf{h}$

household and other twins living in different households, the values suggested that environmental inequities played a role in maintaining large interindividual variations (Table II). However, even this influence of environmental factors on theophylline metabolism did not obscure the prominent role of genetic factors (Table I) in producing large interindividual variations in theophylline kinetics. Because an equal number of $\mathrm{MZ}$ and $\mathrm{DZ}$ twins lived together, the greater environmental contribution to theophylline kinetic variations among twins living apart compared with those living together probably did not influence substantially the high values obtained for $\mathbf{H}_{1}{ }^{2}$.

Distribution curves and probits, pedigree analysis, and Hardy-Weinberg analysis provided evidence for genetic control of interindividual variations in rate constants of formation of each theophylline metabolite. These analyses were all performed on kinetic measurements expressed as rate constants of formation of the three principal metabolites of theophylline.

Distribution curves were constructed from values for $k_{\mathrm{el}}$, $k_{3-\mathrm{MX}}, k_{\mathrm{1}-\mathrm{MU}}$, and $k_{1,3-\mathrm{MU}}$ (Figs. 2-5) obtained from 79 unrelated subjects. Among these 79 subjects, interindividual variations of $k_{\mathrm{el}}$ were fourfold; of 3-MX, sixfold; of 1-MU, eightfold; and of 1,3-MU, sevenfold. Probit regressions, shown in Figs. 2-5, suggested the positions of two antimodes in distribution curves for $k_{3-\mathrm{Mx}}, k_{1-\mathrm{MU}}$, and $k_{1,3-\mathrm{MU}}$. The distribution curve for $k_{\mathrm{el}}$ was difficult to interpret, being compatible with either unimodality or bimodality. Points of inflection in these curves were determined by identifying changes in slopes of the line between probit values of 3 and 7 . Subjects outside of the range from 3 to 7 , although shown, were omitted from consideration in constructing lines since they comprised only a very small 
Table I. Rate Constants of Theophylline Metabolite Formation and Overall Elimination in Six Sets of MZ and Six Sets of DZ Twins

\begin{tabular}{|c|c|c|c|c|}
\hline \multirow[b]{2}{*}{ Subject } & \multicolumn{3}{|c|}{$k_{\mathrm{i}} \mathrm{h}^{-1}\left(\times 10^{-3}\right)$} & \multirow{2}{*}{$\frac{k_{\mathrm{el}} \mathrm{h}^{-1}\left(\times 10^{-2}\right)}{\text { Theophylline }}$} \\
\hline & 3-MX & $1-\mathrm{MU}$ & 1,3-MU & \\
\hline \multicolumn{5}{|c|}{ MZ Twins } \\
\hline LeT & 16.7 & 34.0 & 56.8 & 12.12 \\
\hline $\mathrm{LaT}$ & 20.7 & 37.8 & 59.1 & 11.90 \\
\hline $\mathrm{MiC}$ & 20.7 & 32.0 & 53.1 & 12.47 \\
\hline $\mathrm{MaC}$ & 21.2 & 27.9 & 57.0 & 13.36 \\
\hline TH & 13.2 & 27.6 & 55.3 & 10.66 \\
\hline FH & 10.9 & 30.2 & 57.6 & 9.56 \\
\hline RiD & 5.5 & 10.4 & 23.5 & 8.42 \\
\hline RoD & 4.0 & 5.9 & 21.6 & 7.74 \\
\hline DnM & 7.5 & 12.2 & 32.5 & 7.15 \\
\hline DsM & 8.8 & 14.2 & 36.6 & 10.26 \\
\hline DsL & 15.1 & 17.8 & 44.4 & 8.66 \\
\hline DdL & 11.3 & 19.7 & 50.4 & 9.77 \\
\hline$r$ & 0.90 & 0.68 & 0.98 & 0.72 \\
\hline \multicolumn{5}{|c|}{ DZ Twins } \\
\hline MB & 8.4 & 19.6 & 54.5 & 8.47 \\
\hline DB & 9.1 & 16.0 & 37.7 & 8.08 \\
\hline DT & 17.3 & 32.1 & 69.1 & 13.07 \\
\hline ST & 16.7 & 20.0 & 49.4 & 10.91 \\
\hline $\mathrm{RC}$ & 20.0 & 30.2 & 56.0 & 12.08 \\
\hline DC & 10.4 & 17.3 & 27.6 & 9.04 \\
\hline SnD & 13.0 & 28.2 & 50.0 & 11.64 \\
\hline StD & 15.8 & 25.0 & 61.2 & 11.97 \\
\hline $\mathrm{CH}$ & 15.0 & 21.6 & 41.6 & 9.13 \\
\hline KH & 15.8 & 30.2 & 51.8 & 11.26 \\
\hline CR & 10.6 & 16.0 & 35.4 & 9.20 \\
\hline TR & 10.2 & 17.7 & 29.7 & 7.89 \\
\hline$r$ & 0.37 & 0.07 & 0.16 & 0.49 \\
\hline \multicolumn{5}{|c|}{ Heritability } \\
\hline $\mathrm{H}_{1}^{2}$ & 0.61 & 0.84 & 0.95 & 0.34 \\
\hline $\mathbf{H}_{2}{ }^{2}$ & 0.81 & 0.96 & 0.99 & 0.64 \\
\hline $\mathrm{H}_{3}{ }^{2}$ & 1.0 & 1.0 & 1.0 & 0.47 \\
\hline $\mathrm{H}_{4}{ }^{2}$ & 0.84 & 0.66 & 0.98 & 0.46 \\
\hline
\end{tabular}

$r$, intraclass correlation coefficient.

fraction of the total number of subjects, and thus might be considered misleading if used to construct a separate line (35). Arrows under the probit plots indicate the points of inflection of the line; the arrows below the respective arithmetic distribution curves correspond to these points. Inflection points in the regression for $k_{3-\mathrm{MX}}, k_{1-\mathrm{MU}}$, and $k_{1,3-\mathrm{MU}}$ appeared at antimodes $7.9 \times 10^{-3}$ and $15.5 \times 10^{-3}, 13.0 \times 10^{-3}$ and 23.7
Table II. Intratwin Correlations* of Theophylline Metabolite $k_{i}$ and Also of Theophylline $k_{e l}$ for $M Z$ and $D Z$ Twins Living Together and Apart

\begin{tabular}{lllll}
\hline Parameter & \multicolumn{2}{l}{ Twins living togetherł } & \multicolumn{2}{l}{ Twins living apart§ } \\
\hline & $r$ & $P$ & $r$ & $P$ \\
$k_{\mathrm{el}}$ & 0.77 & $<0.10 \mathrm{NS}$ & 0.27 & $<0.10 \mathrm{NS}$ \\
$k_{3-\mathrm{MX}}$ & 0.97 & $<0.01$ & 0.35 & $<0.10 \mathrm{NS}$ \\
$k_{1-\mathrm{MU}}$ & 0.83 & $<0.05$ & 0.62 & $<0.10 \mathrm{NS}$ \\
$k_{1,3-\mathrm{MU}}$ & 0.78 & $<0.10 \mathrm{NS}$ & 0.26 & $<0.10 \mathrm{NS}$
\end{tabular}

${ }^{*} r=\left(2 \Sigma[Y-\bar{Y}]\left[Y^{\prime}-\bar{Y}^{\prime}\right]\right) /\left(\Sigma[Y-\bar{Y}]^{2}+\Sigma\left[Y^{\prime}-\bar{Y}^{\prime}\right]^{2}\right)$.

$\ddagger$ Three sets each of $M Z$ and $D Z$ twins.

$\S$ Three sets each of $M Z$ and $D Z$ twins.

$\times 10^{-3}$, and $32.0 \times 10^{-3}$ and $47.3 \times 10^{-3}$, respectively, of their distribution curves (Figs. 3-5). It needs to be emphasized that these antimodes are only approximations. Thus, if hundreds of subjects were investigated, the exact position of each antimode would be less precise, so that subjects with values in these overlapping regions of the distribution curve at the antimodes would be difficult to assign as either homozygotes or heterozygotes. Accordingly, errors could arise in distinguishing between phenotypes for subjects in this region.

Table III shows for 27 members of 6 families rate constants of formation of theophylline metabolites 3-MX, 1-MU, and $1,3-\mathrm{MU}$, as well as the overall theophylline $k_{\mathrm{el}}$. Phenotypes for theophylline kinetic constants were determined by the position of each family member's value on the arithmetic distribution curves generated from the 79 unrelated subjects. In turn, these arrows drawn to separate phenotypes on the arithmetic distribution curve were transferred there according to the position of corresponding arrows on the probit regression line (Figs. 3$5)$. Subjects whose phenotypes placed them in the low rate constant range were assigned genotypes $\mathrm{tt}\left(k_{3-\mathrm{MX}}\right), \mathrm{nn}\left(k_{1-\mathrm{MU}}\right)$,

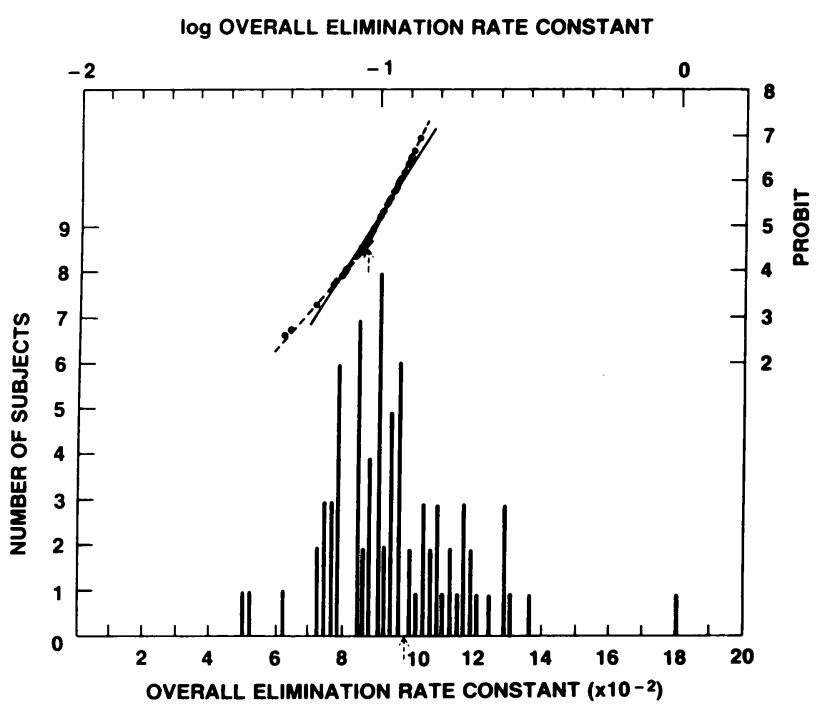

Figure 2. Frequency distribution of theophylline overall elimination rate constants in 79 unrelated subjects. The probit regression appears to be compatible with either a unimodal (solid line) or bimodal (dashed line) distribution. The interrupted arrow indicates the inflection point according to a bimodal interpretation. 


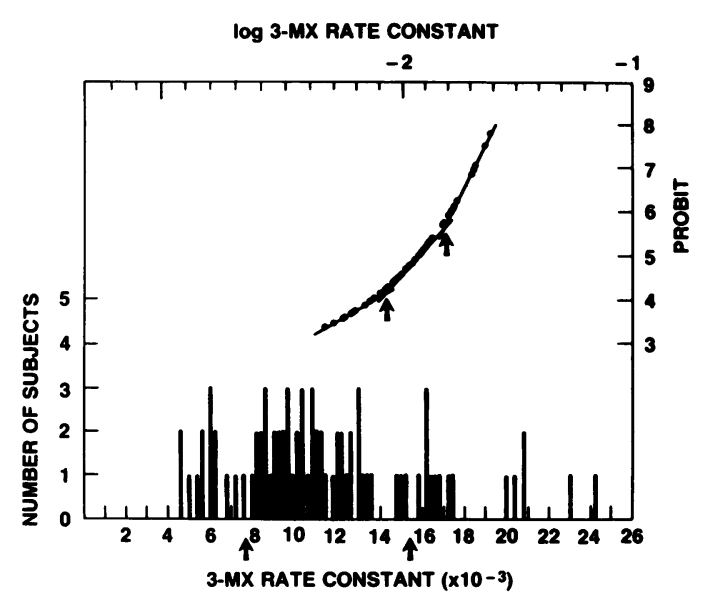

Figure 3. Frequency distribution of rate constants of formation of 3MX in 79 unrelated subjects. Probits are plotted above the frequency distributions. Arrows indicate inflection points. Note that arrows on the probit curve point to log values, and are identical to corresponding arrows on the arithmetic distribution curve.

and dd $\left(k_{1,3-\mathrm{MU}}\right)$. Subjects whose phenotypes fell in the intermediate range were assigned genotypes of $\mathrm{Tt}, \mathrm{Nn}$, and $\mathrm{Dd}$, whereas those in the high range were designated TT, NN, and DD. Alleles that determine low and high rate constants were considered co-dominant, since existence of a middle mode in each distribution curve permitted identification of heterozygotes.

Hardy-Weinberg analysis of genotype frequencies for the 79 unrelated subjects is shown in Table IV. Gene frequencies were $\mathrm{p}=0.49, \mathrm{q}=0.51, \mathrm{p}=0.53, \mathrm{q}=0.47$; and $\mathrm{p}=0.52, \mathrm{q}$ $=0.48$, for $k_{3-\mathrm{MX}}, k_{1-\mathrm{MU}}$, and $k_{1,3-\mathrm{MU}}$, respectively. Observed genotypes did not differ significantly in frequency from HardyWeinberg expectations by chi-square analysis for $k_{1-\mathrm{MU}}$ and $k_{1,3-\mathrm{MU}}$. However, a significant deviation from that expected occurred for $k_{3-\mathrm{Mx}}$ (Table IV).

Fig. 6 shows the six pedigrees. Each pedigree was consistent with a monogenic hypothesis with respect to rate constants of theophylline metabolic formation. However, while for families 1-3 the genotype assignment in almost every subject for one

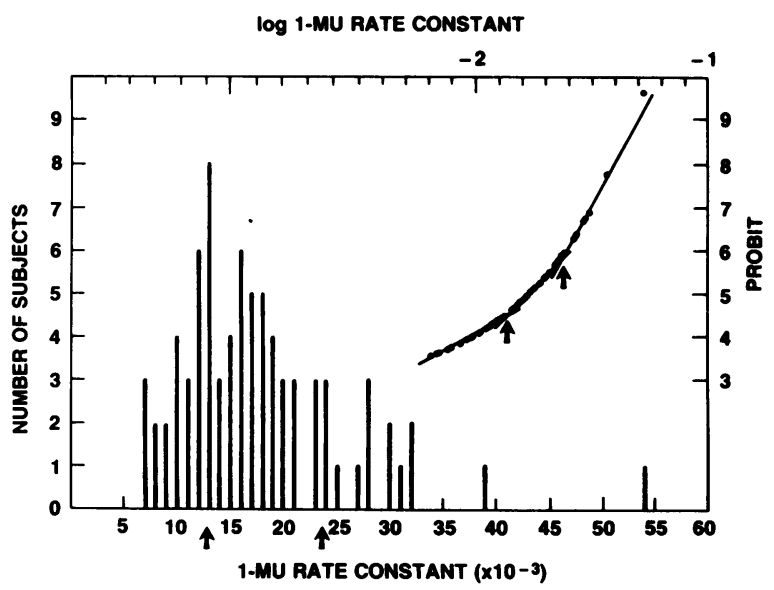

Figure 4. Frequency distribution of rate constants of formation of 1MU in 79 unrelated subjects. Probits are plotted above the frequency distributions. Arrows indicate inflection points. Note that arrows on the probit curve point to log values, and are identical to corresponding arrows on the arithmetic distribution curve.

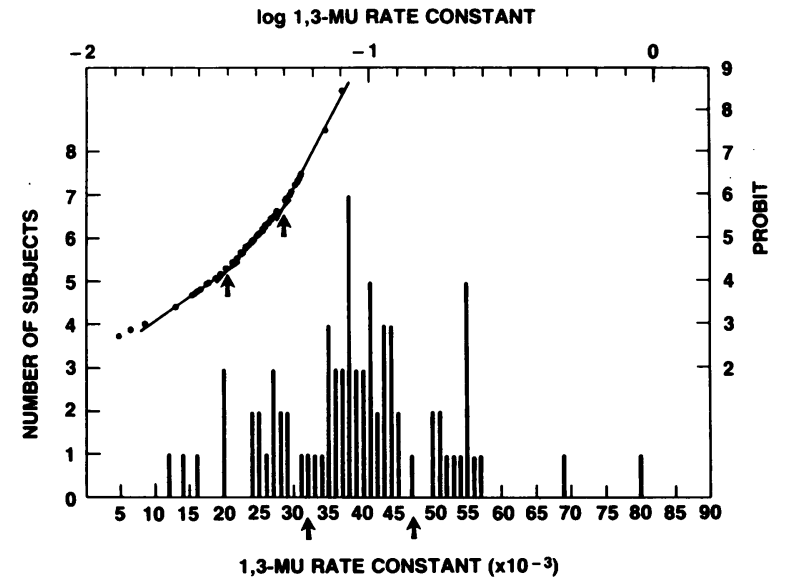

Figure 5. Frequency distribution of rate constants of formation of 1,3-MU in 79 unrelated subjects. Probits are plotted above the frequency distributions. Arrows indicate inflection points. Note that arrows on the probit curve point to $\log$ values, and are identical to corresponding arrows on the arithmetic distribution curve.

theophylline metabolite applied as well for the other two metabolites, in families 4-6 a different genotype had to be assigned for 3-MX from that assigned for 1-MU and 1,3-MU.

Correlation coefficients between rate constants of formation of theophylline metabolites and overall theophylline elimination rate constant in 99 related and unrelated subjects appear in Table V. The mean correlation coefficient for all rate constants was 0.74 . For the three pedigrees in which AP phenotypes had also been determined, Table VI summarizes correlations of theophylline rate constants $\left(k_{\mathrm{el}}, k_{3-\mathrm{MX}}, k_{1-\mathrm{MU}}\right.$, and $\left.k_{1,3-\mathrm{MU}}\right)$ with $\mathrm{AP}$ rate constants (AP $k_{\mathrm{el}}, k_{40 \mathrm{H}-\mathrm{AP}}, k_{30 \mathrm{HM}-\mathrm{AP}}$, and $k_{\mathrm{NDM}-\mathrm{AP}}$ ) (12). Highest correlations occurred between AP $k_{\mathrm{el}}$ and theophylline $k_{\mathrm{el}}, k_{3-\mathrm{MX}}, k_{1-\mathrm{MU}}$, and $k_{1,3-\mathrm{MU}}$. These correlations were 0.77 , $0.69,0.68$, and 0.77 , respectively.

\section{Discussion}

The present investigation illustrates application of a previously proposed method consisting of two separate steps to reveal pharmacokinetic polymorphisms (28): a twin study as an initial screen to determine whether genetic factors are operative in maintaining large interindividual variations and, if so, a family study to test the likelihood of a Mendelian mode of transmission. Our results on twins under near basal environmental conditions suggest that the large interindividual variations in theophylline metabolism that exist even in relatively uninduced subjects arise predominantly from genetic factors (Table I). A fundamental assumption in the use of the twin method to estimate the distinct contributions of genetic and environmental factors to pharmacokinetic variation is that the environments of $\mathrm{MZ}$ and $\mathrm{DZ}$ twins are uniform with respect to critical factors (28). In this study, the pharmacokinetic influence of environmental differences between twins living together and twins living apart was substantial. Potential sources of such environmental differences include various dietary factors (36-40). A higher correlation was observed for all rate constants for twins living together compared to those living apart, regardless of their zygosity, although only the correlations for $k_{3-\mathrm{MS}}(0.97)$ and $k_{1-\mathrm{MU}}(0.83)$ for twins living together were statistically more similar than those living apart 
Table III. Theophylline $k_{e l}$ and Theophylline Metabolite $k_{i}$ in 15 Subjects from 6 Families

\begin{tabular}{|c|c|c|}
\hline & $k_{i} h^{-1}\left(\times 10^{-3}\right)$ & $k_{\mathrm{el}} \mathrm{h}^{-1}\left(\times 10^{-2}\right)$ \\
\hline member & $k_{\text {I-MU }}$ & Theophylline \\
\hline
\end{tabular}

Family 1

$\begin{array}{rrrrr}\mathrm{I}_{1} & 14.0 & 19.1 & 39.5 & 9.37 \\ \mathrm{I}_{2} & 14.8 & 27.7 & 51.0 & 10.95 \\ \mathrm{II}_{1} & 14.0 & 22.4 & 41.8 & 9.78 \\ \mathrm{II}_{2} & 10.7 & 15.3 & 44.3 & 11.03 \\ \mathrm{II}_{3} & 11.6 & 19.7 & 45.7 & 9.81 \\ \mathrm{II}_{4} & 17.1 & 27.6 & 54.4 & 11.15\end{array}$

Family 2

$\begin{array}{rr}\mathrm{I}_{1} & 13.5 \\ \mathrm{I}_{2} & 12 . \\ \mathrm{II}_{1} & 12.4 \\ \mathrm{II}_{2} & 6 .\end{array}$

\section{5}

12.1

12.4

6.3

\section{5}

21.3

15.1

12.8

\section{3}

43.0

46.0

27.1

9.36

9.01

9.26

7.68

\section{Family 3}

$\begin{array}{lr}\mathrm{I}_{1} & 9 \\ \mathrm{I}_{2} & 24 \\ \mathrm{II}_{1} & 16.7 \\ \mathrm{II}_{2} & 20.7 \\ \mathrm{II}_{3} & 12.9\end{array}$

Family 4

$I_{1}$

$I_{2}$

9.2

22.7

38.7

80.1

8.70

53.5

34.2

37.8

56.8

18.02

12.12

11.90

35.2

9.99

19.6

13.35

37.8

17.0

8.9

17.8

11.3

28.9

9.00

36.1

12.55

$\mathrm{II}_{2}$

12.4

Family 5

$I_{1}$

$I_{2}$

19.0
13.3
17.3
16.9

18.8

12.0

37.0

22.6

10.27

17.3

30.1

7.74

8.90

$\mathrm{II}_{2}$

14.8

28.2

9.57

Family 6

\begin{tabular}{rrrrr}
$\mathrm{I}_{1}$ & 12.6 & 7.5 & 16.2 & 7.70 \\
$\mathrm{I}_{2}$ & 21.0 & 18.9 & 34.4 & 10.16 \\
$\mathrm{II}_{1}$ & 20.0 & 18.3 & 25.0 & 7.97 \\
$\mathrm{II}_{2}$ & 21.9 & 15.3 & 25.9 & 8.72 \\
\hline
\end{tabular}

Table IV. Hardy-Weinberg Analysis of Genotype Frequencies for Theophylline Metabolite $k_{i}$ in 79 Unrelated Subjects

\begin{tabular}{lllllll}
\hline $\begin{array}{l}\text { Rate } \\
\text { constant }\end{array}$ & $\begin{array}{l}\text { Geno- } \\
\text { type }\end{array}$ & $\begin{array}{l}\text { Observed } \\
(\mathrm{O})\end{array}$ & $\begin{array}{l}\text { Expected } \\
(\mathrm{E})\end{array}$ & $\frac{(\mathrm{O}-\mathrm{E})^{2}}{\mathrm{E}}$ & $\begin{array}{l}\text { Chi-Square } \\
\mathrm{df}=1, n=79\end{array}$ \\
\hline$k_{3-\mathrm{Mx}}$ & $\mathrm{tt}$ & 15 & 19 & 0.84 & & \\
& $\mathrm{Tt}$ & 49 & 39 & 2.56 & 5.11 & $\mathrm{~S}$ \\
& $\mathrm{TT}$ & 15 & 21 & 1.71 & & \\
$k_{1-\mathrm{MU}}$ & $\mathrm{nn}$ & 20 & 22 & 0.18 & & $\mathrm{NS}$ \\
& $\mathrm{Nn}$ & 44 & 39 & 0.64 & 1.32 & \\
& $\mathrm{NN}$ & 15 & 18 & 0.50 & & \\
$k_{1,3-\mathrm{MU}}$ & $\mathrm{dd}$ & 20 & 21 & 0.05 & & $\mathrm{NS}$ \\
& $\mathrm{Dd}$ & 42 & 40 & 0.10 & 0.21 &
\end{tabular}

For gene frequencies, $p=t, n, d ; q=T, N, D . S$, significant.
( 0.35 and 0.62 , respectively). However, even the heterogeneous environmental conditions present in this study could not conceal the large role played by genetic factors in the regulation of hepatic production of the three major theophylline metabolites. This large genetic role was indicated by the high values for heritability $\left(\mathrm{H}_{1}{ }^{2}\right)$ of variations in the rate constants of formation of the three principal theophylline metabolites (Table I). In our study, fortunately, 3 sets of $M Z$ and 3 sets of $D Z$ twins lived together and three sets of $\mathrm{MZ}$ and three sets of $\mathrm{DZ}$ twins lived apart. This equality prevented the environmental differences discussed above from exerting a major influence on estimates of $\mathbf{H}^{2}$.

While use of twins revealed that genetic factors control phenotypic pharmacokinetic variations in theophylline disposition, pedigree analysis was required to establish or reject a Mendelian mode of transmission of these genetic factors. Pedigree analysis in six families and the trimodal distribution curves in the 79 unrelated subjects support a monogenic hypothesis for the control of the sixfold to eightfold interindividual variations in rate constants of formation of the three main theophylline metabolites. The alleles are transmitted as autosomal co-dominants.

It is uncertain whether more than one genetic polymorphism controls these interindividual variations in rate constants of formation of 3-MX, 1-MU, and 1,3-MU. An interpretation that only one polymorphism regulates formation of all three metabolites is supported by high, statistically significant correlations among these theophylline metabolite rate constants (Table V). Thus, a common gene locus may either directly control, or at least participate in the regulation of all 3 oxidation reactions. Additional evidence for the regulation of all rate constants of formation of theophylline metabolites by a single gene locus includes the similar gene frequencies for rate constants of formation of each theophylline metabolite (Table IV), the trimodality of the distribution and probit curves (Figs. 3-5), and some aspects of the pedigree analyses (Fig. 6), particularly genotype assignments for families 1-3, where the genotype assignment of almost every subject for one metabolite applies equally well to the two other metabolites. However, for families 4-6, genotype assignment that applied in a subject for both 1-MU and 1,3-MU changed for 3-MX, which is an observation difficult to explain if there is a common gene for all three metabolites. Present concepts in drug metabolism hold that individual oxidative pathways for many drugs are catalyzed not by a single cytochrome P-450, but rather by a family of cytochrome P-450 isozymes. Thus, more than one genetic polymorphism and cytochrome P-450 isozyme may participate in theophylline metabolism. Participation of several such distinct genetic polymorphisms and cytochrome P-450 isozymes in theophylline metabolism is also supported by distribution curves that differ for theophylline $k_{\mathrm{el}}$ and the three metabolites, as well as by developmental and enzyme induction studies revealing genetically distinct pathways of theophylline metabolism. These studies indicate that $\mathrm{N}$ demethylation and 8-hydroxylation of theophylline are mediated by two separate forms of cytochrome P-450 (3, 13-15).

It seemed reasonable that other drugs, such as $\mathrm{AP}$, that also exhibit genetic polymorphism (12) as well as metabolism by $\mathrm{N}$-demethylation and hydroxylation reactions, might share with theophylline certain biotransforming pathways and hence genetic regulation. The possibility of common, shared cytochrome P-450 isozymes that participate in the metabolism of 
Rate Constant of Formation for 3-MX
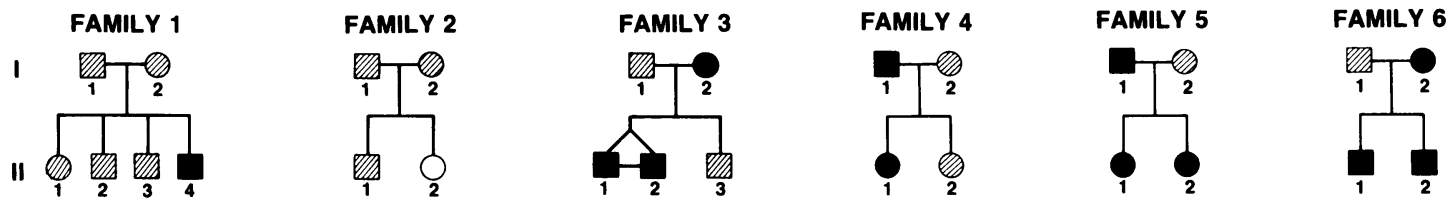

Rate Constant of Formation for 1-MU
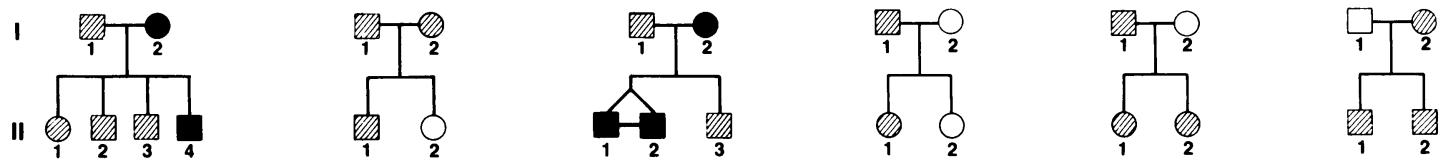

Rate Constant of Formation for 1,3-MU
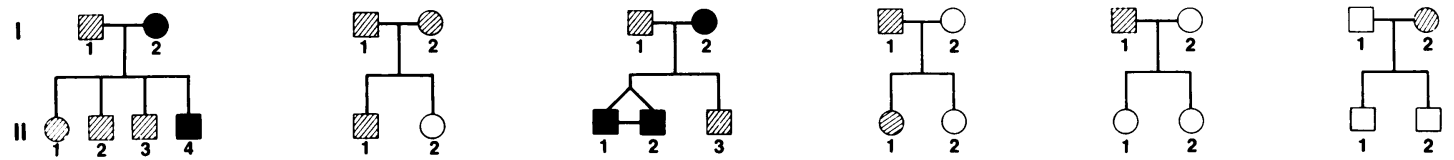

Homozygous low

Heterozygous

Homozygous high

Figure 6. Six pedigrees showing transmission of phenotypes for rate constants of formation of 3-MX, 1-MU, and 1,3MU. All pedigrees are consistent with a monogenic hypothesis.

different drugs has important therapeutic implications, since genetically fast or slow metabolizers of theophylline could then be predicted to biotransform related drugs in a similar manner. This approach of studying a panel of different drugs in pharmacogenetically well-defined subjects has been particularly useful in identifying the pharmacokinetic consequences of the genetic locus that controls the metabolism of debrisoquine and 22 other drugs (6).

By contrast, the modality of the distribution curve and probit plot for theophylline $k_{\mathrm{el}}$ is difficult to interpret, probably because $k_{\mathrm{el}}$ is a more distant reflection of the genes under investigation than the rate constant of formation of each theophylline metabolite. In addition to contributions from each of the three rate constants of theophylline metabolite formation, the value of theophylline $k_{\mathrm{el}}$ incorporates effects not only from other unmeasured theophylline metabolites but also renal excretion of theophylline itself. Accordingly, the distribution curve and probit plot of theophylline $k_{\mathrm{el}}$ are

Table V. Correlations between Theophylline Rate Constants* in 99 Related and Unrelated Subjects

\begin{tabular}{lll}
\hline \multicolumn{1}{c}{ Rate constants compared } & \multicolumn{2}{l}{ Correlation coefficient } \\
\hline & $r$ & $P$ \\
$k_{\text {el }}-k_{3-\mathrm{MX}}$ & 0.77 & $<0.001$ \\
$k_{\mathrm{el}}-k_{1-\mathrm{MU}}$ & 0.75 & $<0.001$ \\
$k_{\mathrm{el}}-k_{1,3-\mathrm{MU}}$ & 0.79 & $<0.001$ \\
$k_{3-\mathrm{MX}}-k_{1-\mathrm{MU}}$ & 0.74 & $<0.001$ \\
$k_{3-\mathrm{MX}}-k_{1,3-\mathrm{MU}}$ & 0.67 & $<0.001$ \\
$k_{1-\mathrm{MU}}-k_{1,3-\mathrm{MU}}$ & 0.76 & $<0.001$ \\
\hline
\end{tabular}

${ }^{*} k_{\mathrm{el}}, k_{3-\mathrm{MX}}, k_{1-\mathrm{MU}}$, and $k_{1,3-\mathrm{MU}}$. unclear and consistent with either unimodality or bimodality. While several separate cytochrome P-450 isozymes involved in theophylline metabolism may share a common regulatory locus, these isozymes are differentially susceptible to other factors of a developmental and environmental nature $(14,15$, $41,42)$. Such factors exerting unequal effects on the different isozymes of cytochrome P-450 could account not only for the correlations in Table $\mathrm{V}$ not being higher but also for the equivocal distribution curve of theophylline $k_{\mathrm{el}}$. These considerations collectively emphasize the need to select for pharmacogenetic investigation measurements closer to the gene products than just elimination rates of parent drugs.

Hardy-Weinberg analysis of genotype frequencies for the 79 unrelated subjects (Table IV) suggests almost equal frequencies of the postulated alleles $p$ and $q$ that appear to control high and low values for rate constants of each theophylline metabolite. Only for $k_{3-\mathrm{Mx}}$ did chi-square analysis show a significant departure of observed from expected phenotype. This is not surprising and could be due to the small number of subjects studied or to nonrandom mating. The remote possibility of a selective advantage of the heterozygote cannot be eliminated.

The results of these studies on theophylline metabolism are compatible with those recently published by Miller et al. (9). Nevertheless, several methodological differences between these studies exist. Their twin study on causes of large interindividual variations in theophylline elimination presented no evidence to suggest a commonly occurring polymorphism of theophylline metabolism. On the contrary, the single pedigree is offered as a possible example of a very rare genetic variation. Many subjects in the study of Miller et al. (9) were enzymatically induced through smoking. In our study, use of rate constants measured in carefully selected twins and families under near 
Table VI. Coefficients of Correlation between $k_{e l}$ and $k_{i}$ from Metabolic Data on AP and Theophylline Administered at Different Times to 15 Subjects from 3 Families

\begin{tabular}{|c|c|c|c|c|c|c|c|c|}
\hline & \multicolumn{2}{|c|}{ Theophylline } & \multirow[b]{2}{*}{$k_{3-M U}$} & & \multirow[b]{2}{*}{$k_{1-\mathrm{MU}}$} & & \multirow[b]{2}{*}{$k_{1,3-\mathrm{MU}}$} & \\
\hline & $k_{\text {d }}$ & & & & & & & \\
\hline & & $P$ & & $P$ & & $P$ & & $P$ \\
\hline $\mathrm{AP} k_{\mathrm{el}}$ & 0.77 & $<0.01$ & 0.69 & $<0.01$ & 0.68 & $<0.01$ & 0.77 & $<0.01$ \\
\hline$k_{4 \mathrm{OH}-\mathrm{AP}}$ & 0.02 & $<0.10 \mathrm{NS}$ & 0.03 & $<0.10 \mathrm{NS}$ & 0.09 & $<0.10 \mathrm{NS}$ & 0.00 & $<0.10 \mathrm{NS}$ \\
\hline$k_{3 \mathrm{OH}-\mathrm{AP}}$ & 0.15 & $<0.10 \mathrm{NS}$ & 0.27 & $<0.10 \mathrm{NS}$ & 0.26 & $<0.10 \mathrm{NS}$ & 0.28 & $<0.10 \mathrm{NS}$ \\
\hline$k_{\text {NDP-AP }}$ & 0.04 & $<0.10 \mathrm{NS}$ & 0.05 & $<0.10 \mathrm{NS}$ & 0.18 & $<0.10 \mathrm{NS}$ & 0.01 & $<0.10 \mathrm{NS}$ \\
\hline
\end{tabular}

basal environmental conditions probably explains why our values of heritability were higher than those of Miller et al. (9). The single pedigree presented by Miller et al. (9) was difficult to interpret genetically due to lack of distribution curves in unrelated subjects according to which the members of their family could be phenotyped. Possibly, that mating involved a rare homozygote and a heterozygote for a different mutation from the commonly occurring one under investigation here. Finally, in contrast to other investigations in adults (19, 20), Miller et al. (9) reported sex differences in theophylline metabolism. In the present study of 99 subjects, no sex differences occurred in any of the kinetic measurements.

The previously reported genetic polymorphism of AP (12) appears to be related to that of theophylline, since in the 15 family members studied with both drugs, significant correlations occurred between AP $k_{\mathrm{el}}$ and theophylline $k_{\mathrm{el}}, k_{3-\mathrm{MX}}, k_{1-\mathrm{MU}}$, and $k_{1,3-\mathrm{MU}}$ (Table VI). These correlations suggest a common underlying genetic factor regulating theophylline and AP metabolism. However, the low correlations between rate constants of formation of theophylline and AP metabolites and also between individual metabolites of AP and theophylline $k_{\mathrm{el}}$ suggest that while the pathways for AP and theophylline disposition may share some common regulatory factor, several cytochrome P-450 isozymes responsible for producing AP and theophylline metabolites may differ. This latter possibility is supported by the different gene frequencies of the AP polymorphism where $q$ varies between 0.1 and 0.2 for the three main AP metabolites and the theophylline polymorphism where $\mathrm{q}$ approximates 0.5 for each metabolite. All these considerations indicate that even in normal subjects under carefully regulated environmental conditions, control of large interindividual variations in the metabolism of AP and theophylline is complex.

Other investigators have also reported high correlations among subjects between their rate of theophylline and AP metabolism. Teunissen et al. (17) studied the correlation between AP and theophylline metabolite formation in man and concluded that total theophylline metabolism and the formation of $40 \mathrm{H}-\mathrm{AP}$ are mediated by closely related, or even the same isozymes of cytochrome P-450. Inhibitors of drug metabolism such as cimetidine impair to similar extents the elimination of both theophylline and AP (43-45). A diet high in charcoal-broiled beef induces to similar extents the metabolism of AP and theophylline (37), and high dietary protein can by itself induce both antipyrine and theophylline (39). Finally, cigarette smoking induces to similar extents the metabolism of AP and theophylline $(14,19,20,41,42,46-48)$. All these parallel changes in rates of AP and theophylline metabolism are consistent with similar metaboic regulation.
Because rates of theophylline and AP metabolism are correlated in a subject, and because it has been demonstrated that the AP polymorphism differs from the polymorphism of debrisoquine oxidation (48-50), the theophylline polymorphism is probably unrelated to that of debrisoquine. In agreement with this conclusion, Dahlqvist et al. (18) reported that no relationship occurred between rates of theophylline or antipyrine metabolism in a subject, on the one hand, and of debrisoquine or sparteine on the other. This result suggests that the hepatic cytochrome P-450 isozymes that biotransform theophylline and AP differ from those that metabolize debrisoquine and sparteine.

Since interpretation of these and previously published pharmacogenetic studies relies heavily on correlations between rate constants of formation of different metabolites, the caution and pitfalls emphasized earlier (12) as inherent in such interpretations should be restated. Some pharmacokinetic correlations, such as those in Table $\mathrm{V}$, while only moderately strong $(r=0.7-0.8)$, were highly statistically significant $(P<0.001)$ because of the relatively large number (99) of subjects investigated. Confusion occasionally arises between the numerical value of a correlation coefficient and the predictability of that correlation. They are not synonymous: the predictability of one variable based on its correlation to another is not determined by $r$ but by $r^{2}$ (51), a much lower number than $r$. The widespread practice of using correlations as proof of a pharmacological relationship, rather than as a clue to design and perform a well-controlled experiment that adequately tests a hypothesis, should be discouraged $(52,53)$.

Depending on the drug selected for study and the population(s) investigated, large variations, ranging from 4-fold to 40-fold, occur among subjects for many other commonly prescribed drugs $(28,29,54,55)$. If uncompensated by dosage adjustment, these large pharmacokinetic variations can produce toxicity in patients who metabolize a drug-like theophylline slowly and undertherapy in other patients who metabolize theophylline more rapidly than normal. Risks of toxicity in genetically determined slow metabolizers are enhanced for a drug like theophylline because therapeutic doses can saturate hepatic biotransforming capacity $(4,22,34)$ which could produce exponential increases in serum theophylline concentrations. A convenient method to discover pharmacogenetic conditions (28) uses a tier system, which begins with screening in twins to determine whether a genetic factor exists. If so, investigations proceed to carefully selected families who were drawn from propositi with unusual values. Clearcut segregation patterns of these unusual phenotypes reveal their Mendelian mode of transmission (28). This method has been applied previously to identify the polymorphism affecting AP (12). 


\section{Acknowledgments}

The authors gratefully acknowledge the laboratory assistance of Cheryl L. Denlinger and Dr. Karen A. Powers.

\section{References}

1. Hendeles, L., and M. Weinberger. 1983. Theophylline: a "state of the art" review. Pharmacotherapy. 3:2-44.

2. Jenne, J. W., E. Wyze, F. S. Rood, and F. M. MacDonald. 1972. Pharmacokinetics of theophylline: application to adjustment of the clinical dose of aminophylline. Clin. Pharmacol. Ther. 13:349360 .

3. Ginchansky, E., and M. Weinberger. 1977. Relationship of theophylline clearance to oral dosage in children with chronic asthma. J. Pediatr. 91:655-660.

4. Weinberger, M., and E. Ginchansky. 1977. Dose-dependent kinetics of theophylline disposition in asthmatic children. J. Pediatr. 91:820-824.

5. Ellis, E. F., R. Koysooko, and G. Levy. 1976. Pharmacokinetics of theophylline in children with asthma. Pediatrics. 58:542-547.

6. Idle, J. R., and R. L. Smith. 1979. Polymorphisms of oxidation at carbon centers of drugs and their clinical significance. Drug Metab. Rev. 9:301-317.

7. Riegelman, S., K. Muir, and R. Upton. 1980. Factors affecting the pharmacokinetics of theophylline. Eur. J. Respir. Dis. 61(Suppl. 109):67-82.

8. Ogilvie, R. I. 1978. Clinical pharmacokinetics of theophylline. Clin. Pharmacokinet. 3:267-293.

9. Miller, M., K. E. Opheim, V. A. Raisys, and A. G. Motulsky. 1984. Theophylline metabolism: variation and genetics. Clin. Pharmacol. Ther. 35:170-182.

10. Mahgoub, A., J. R. Idle, L. G. Dring, R. Lancaster, and R. L. Smith. 1977. Polymorphic hydroxylation of debrisoquine in man. Lancet. II:584-586.

11. Kupfer, A., P. Desmond, S. Schenker, and R. Branch. 1979. Family study of a genetically determined deficiency of mephenytoin hydroxylation in man. Pharmacologist. 21:173.

12. Penno, M. B., and E. S. Vesell. 1983. Monogenic control of variations in antipyrine metabolite formation: new polymorphism of hepatic drug oxidation. J. Clin. Invest. 71:1698-1709.

13. Lohmann, S. M., and R. P. Miech. 1976. Theophylline metabolism by the rat liver microsomal system. J. Pharmacol. Exp. Ther. 196:213-225.

14. Grygiel, J. J., and D. J. Birkett. 1980. Effect of age on patterns of theophylline metabolism. Clin. Pharmacol. Ther. 28:456-462.

15. Tserng, K., K. C. King, and F. N. Takieddine. 1981. Theophylline metabolism in premature infants. Clin. Pharmacol. Ther. 29: 594-600.

16. Penno, M. B., B. H. Dvorchik, and E. S. Vesell. 1981. Genetic variation in rates of antipyrine metabolite formation: a study in uninduced twins. Proc. Natl. Acad. Sci. USA. 78:5193-5196.

17. Teunissen, M. 1984. Factors and conditions affecting antipyrine clearance and metabolite formation. Ph.D. thesis. University of Leiden, Holland. 215 pp.

18. Dahlqvist, R., L. Bertilsson, D. J. Birkett, M. Eichelbaum, J. Sawe, and F. Sjoqvist. 1984. Theophylline metabolism in relation to antipyrine, debrisoquine and sparteine metabolism. Clin. Pharmacol. Ther. 35:815-821.

19. Jusko, W. J., M. J. Gardner, A. Mangione, J. J. Schentag, J. R. Koup, and J. W. Vance. 1979. Factors affecting theophylline clearances: age, tobacco, marijuana, cirrhosis, congestive heart failure, obesity, oral contraceptives, benzodiazepines, barbiturates, and ethanol. J. Pharm. Sci. 68:1358-1366.

20. Powell, J. R., J. Thiercelin, S. Vozeh, L. Sansom, and S.
Riegelman. 1977. The influence of cigarette smoking and sex on theophylline disposition. Am. Rev. Respir. Dis. 116:17-23.

21. Muir, K. T., J. H. G. Jonkman, D. Tang, M. Kunitani, and S. Riegelman. 1980. Simultaneous determination of theophylline and its major metabolites in urine by reversed-phase ion-pair high-performance liquid chromatography. J. Chromatogr. 221:85-95.

22. Tang-Liu, D. D., R. L. Williams, and S. Riegelman. 1982. Nonlinear theophylline elimination. Clin. Pharmcol. Ther. 31:358369.

23. Upton, R. A., L. Sansom, R. W. Guentert, J. R. Powell, J. Thiercelin, V. P. Shah, P. E. Coates, and S. Riegelman. 1980. Evaluation of the absorption from 15 commercial theophylline products indicating deficiencies in currently applied bioavailability criteria. J. Pharmacokinet. Biopharm. 8:229-242.

24. Rovei, V., F. Chanoine, and M. S. Benedetti. 1982. Pharmacokinetics of theophylline: a dose-range study. Br. J. Clin. Phramacol. 14:769-778.

25. Dvorchik, B. H., and E. S. Vesell. 1978. Significance of error associated with use of the one-compartment formula to calculate clearance of thirty-eight drugs. Clin. Pharmcol. Ther. 23:617-623.

26. Gibaldi, M., and D. Perrier. 1982. Pharmacokinetics. Marcel Dekker, New York. 494 pp.

27. Notari, R. E. 1980. Biopharmaceutics and Clinical Pharmacokinetics. Marcell Dekker, New York. Third ed. 1-388.

28. Vesell, E. S. 1978. Twin studies in pharmacogenetics. Hum. Genet. 1(Suppl.):19-30.

29. Vesell, E. S. 1973. Advances in pharmacogenetics. In Progress in Medical Genetics, IX. A. G. Steinberg and A. G. Bearn, editors. Grune and Stratton Inc., New York. 291-367.

30. Vogel, F., and A. G. Motulsky. 1979. Heritability estimates from twin data. In Human Genetics: Problems and Approaches. Springer-Verlag, Berlin. 586-590.

31. Steel, R. G. D., and J. H. Torrie. 1980. Homogeneity of correlation coefficients. Principles and Procedures of Statistics: A Biometric Approach. McGraw-Hill, Inc., New York. 283-284.

32. Burn, J. H., D. J. Finney, and L. G. Goodwin. 1952. Classification of methods. Biological Standardization. Oxford University Press, Oxford. 2:8-25.

33. Monks, T. J., J. Caldwell, and R. L. Smith. 1979. Influence of methylxanthine-containing foods on theophylline metabolism and kinetics. Clin. Pharmcol. Ther. 26:513-524.

34. Gundert-Remy, U., R. Hildebrandt, N. Hengen, and E. Weber. 1983. Nonlinear elimination processes of theophylline. Eur. J. Clin. Pharmacol. 24:71-78.

35. Goldstein, A. 1964. Correlation. Biostatistics: An Introductory Text. MacMillan Publishing Co., New York. 4:129-231.

36. Anderson, K. E., A. H. Conney, and A. Kappas. 1982. Nutritional influences on chemical biotransformations in humans. Nutr. Rev. 40:161-171.

37. Kappas, A., A. P. Alvares, K. E. Anderson, E. J. Pantuck, C. B. Pantuck, R. Chang, and A. H. Conney. 1978. Effect of charcoalbroiled beef on antipyrine and theophylline metabolism. Clin. Pharmcol. Ther. 23:445-450.

38. Pantuck, E. J., C. B. Pantuck, W. A. Garland, B. H. Min, L. W. Wattenberg, K. E. Anderson, A. Kappas, and A. H. Conney. 1979. Stimulatory effect of brussels sprouts and cabbage on human drug metabolism. Clin. Pharmacol. Ther. 25:88-95.

39. Kappas, A., K. E. Anderson, A. H. Conney, and A. P. Alvares. 1976. Influence of dietary protein and carbohydrate on antipyrine and theophylline metabolism in man. Clin. Pharmacol. Ther. 20:643-653.

40. Feldman, C. H., V. E. Hutchinson, C. E. Pippenger, T. A. Blumenfeld, B. R. Feldman, and W. J. Davis. 1980. Effect of dietary protein and carbohydrate on theophylline metabolism in children. Pediatrics. 66:956-962.

41. Grygiel, J. J., and D. J. Birkett. 1981. Cigarette smoking and theophylline clearance and metabolism. Clin. Pharmacol. Ther. 30: 491-496. 
42. Bonati, M., R. Latini, G. Marra, B. M. Assael, and R. Parini. 1981. Theophylline metabolism during the first month of life and development. Pediatr. Res. 15:304-308.

43. Lalonde, R. L., R. A. Koob, W. M. McLean, and A. J. Balsys. 1983. The effects of cimetidine on theophylline pharmacokinetics at steady state. Chest. 83:221-224.

44. Slusher, L. B., and E. S. Vesell. 1984. Cimetidine-induced reductions in gastrointestinal absorption of antipyrine and rate constants for formation of its metabolites. Clin. Pharmcol. Ther. 35:568-575.

45. Roberts, R. K., J. Grice, L. Wood, V. Petroff, and C. McGuffie. 1981. Cimetidine impairs the elimination of theophylline and antipyrine. Gastroenterology. 81:19-21.

46. Gardner, M. J., K. M. Tornatore, W. J. Jusko, and R. Kanarkowski. 1983. Effects of tobacco smoking and oral contraceptive use on theophylline disposition. Br. J. Clin. Pharmacol. 16:271-280.

47. Vestal, R. E., A. H. Norris, J. D. Tobin, B. H. Cohen, N. W. Shock, and R. Andres. 1975. Antipyrine metabolism in man: influence of age, alcohol, caffeine, and smoking. Clin. Pharmacol. Ther. 18:425432.

48. Eichelbaum, M., L. Bertilsson, and J. Sawe. 1983. Antipyrine metabolism in relation to polymorphic oxidations of sparteine and debrisoquine. Br. J. Clin. Pharmacol. 15:317-321.
49. Danhof, M., D. D. Breimer, J. R. Idle, T. Sloan, and R. L. Smith. 1980. Antipyrine metabolite formation in extensive and poor metabolizers of debrisoquine. World Conference on Clinical Pharmacology and Therapeutics. The MacMillan Co., London and BasingStoke. 0865. (Abstr.)

50. Bertilsson, L., M. Eichelbaum, B. Mellstrom, J. Sawe, H. U. Schulz, and F. Sjoqvist. 1980. Nortriptyline and antipyrine clearance in relation to debrisoquine hydroxylation in man. Life Sci. 27:16731677.

51. Atlas, S. A., E. S. Vesell, and D. N. Nebert. 1976. Genetic control of interindividual variations in the inducibility of arylhydrocarbon hydroxylase in cultured human lymphocytes. Cancer Res. 36: 4619-4630.

52. Vesell, E. S. 1979. The antipyrine test in clinical pharmacology: conceptions and misconceptions. Clin. Pharmacol. Ther. 26:275-286.

53. Vesell, E. S., and M. B. Penno. 1983. Assessment of methods to identify sources of interindividual pharmacokinetic variations. Clin. Pharmacokinet. 8:378-409.

54. Levy, G., and R. Koysooko. 1976. Renal clearance of theophylline in man. J. Clin. Pharmacol. 16:329-332.

55. Dvorchik, B. H., and E. S. Vesell. 1976. Pharmacokinetic interpretation of data gathered during therapeutic drug monitoring. Clin. Chem. 22:868-878. 